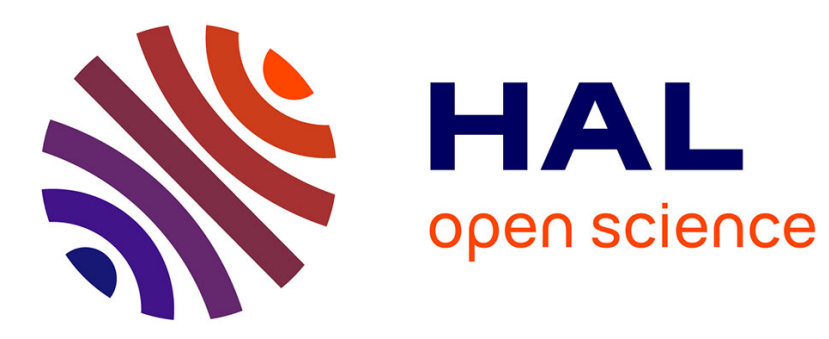

\title{
Boron and nitrogen codoping effect on transport properties of carbon nanotubes
}

\author{
R. Zoubkoff, F. Triozon, Y.-M. Niquet, S. Latil
}

\section{To cite this version:}

R. Zoubkoff, F. Triozon, Y.-M. Niquet, S. Latil. Boron and nitrogen codoping effect on transport properties of carbon nanotubes. Physical Review B: Condensed Matter and Materials Physics (19982015), 2014, 90, pp.125418. 10.1103/PhysRevB.90.125418 . cea-01376289

\section{HAL Id: cea-01376289 https://hal-cea.archives-ouvertes.fr/cea-01376289}

Submitted on 4 Oct 2016

HAL is a multi-disciplinary open access archive for the deposit and dissemination of scientific research documents, whether they are published or not. The documents may come from teaching and research institutions in France or abroad, or from public or private research centers.
L'archive ouverte pluridisciplinaire HAL, est destinée au dépôt et à la diffusion de documents scientifiques de niveau recherche, publiés ou non, émanant des établissements d'enseignement et de recherche français ou étrangers, des laboratoires publics ou privés. 


\title{
Boron and nitrogen codoping effect on transport properties of carbon nanotubes
}

\author{
R. Zoubkoff, ${ }^{1,2}$ F. Triozon, ${ }^{3}$ Y.-M. Niquet, ${ }^{4}$ and S. Latil ${ }^{2, *}$ \\ ${ }^{1}$ COMP Centre of Excellence, Department of Applied Physics, Aalto University, P.O. Box 11100, 00076 Aalto, Finland \\ ${ }^{2}$ CEA, IRAMIS, SPEC, GMT, 91191 Gif-sur-Yvette, France \\ ${ }^{3}$ CEA, LETI, MINATEC, 38054 Grenoble, France \\ ${ }^{4} L \_$Sim, SP2M, UMR-E CEA/UJF-Grenoble 1, INAC, Grenoble, France
}

(Received 12 February 2014; revised manuscript received 28 July 2014; published 11 September 2014)

\begin{abstract}
This paper reports a theoretical study of the effect of boron and nitrogen codoping on the transport properties of carbon nanotubes (CNTs) at the mesoscopic scale. A new tight-binding parametrization has been set up, based on density functional theory calculations, that enables a reliable description of the electronic structure of realistic BN-doped CNTs. With this model, we have carried out a deep analysis of the electronic mean free path (MFP) exhibited by these nanostructures. The MFP is highly sensitive to the geometry of the scattering centers. We report that the relative distance between $\mathrm{B}$ and $\mathrm{N}$ atoms in the network influences drastically the electronic conduction. Moreover, we point out that the scattering induced by small hexagonal BN domains in the carbon network is less important than the BN-pair case.
\end{abstract}

DOI: 10.1103/PhysRevB.90.125418

PACS number(s): 61.48.De, 73.22.-f, 73.23.-b, 31.15.aq

\section{INTRODUCTION}

Single-wall carbon nanotubes (CNTs) have attracted an intense research effort from both fundamental and applied aspects. They exhibit very promising electronic properties that are directly related to their specific quasi-one-dimensional geometry. In order to tune their electronic structure and properties, $\mathrm{B}$ and $\mathrm{N}$ chemical doping can be used. The substitutional doping of $\mathrm{B}$ and/or $\mathrm{N}$ onto the hexagonal network has been intensively studied [1].

Beyond doping, the direct synthesis of C-BN heterostructures is highly desirable, because nanotubes made from hexagonal boron nitride (h-BN) are wide gap insulators [2]. We expect that C-BN heterojunctions can act as basic nanodevices, since the electronic properties of selected regions can be tailored to act as $n$ or $p$ semiconductors. Many applications can be addressed in nanoelectronics, nonlinear optics, or field emission sources. Doped carbon CNTs can be produced by adding moieties containing $\mathrm{B}$ or $\mathrm{N}$ elements during the growth process $[3,4]$. They can also be produced by a high-temperature chemical posttreatment that includes heteroatoms onto the carbon sheet [5].

The chemical nature and the specific geometry induced by the substitutional sites have been probed by various techniques: electronic energy loss spectroscopy, x-ray photoemission spectroscopy, and scanning tunnelling microscopy. More recently, nanotube C-BN heterostructures have been synthesized [6]. In these nanostructures, boron and nitrogen species are known to form hexagonal $\mathrm{BN}$ domains embedded into the carbon network.

In parallel to these experimental investigations on growth and characterization, theoretical studies have been carried out on the $\mathrm{BN}$ domain geometrical structures and on the electronic transport properties of $\mathrm{B}-$ or $\mathrm{N}$-doped nanotubes $[7,8]$. For a single substitution in the carbon honeycomb lattice, it was established that the transmission function presents minimum

\footnotetext{
*sylvain.latil@cea.fr
}

values corresponding to the quasibound state (QBS) induced by the dopants [7]. Conductance is damped for injection energy around $0.5 \mathrm{eV}$ above (below) the Fermi level $E_{F}$ in the case of $\mathrm{N}$ (B) substitution. The QBSs affect the electronic conduction in broader ranges when distributions of dopants are taken into account. The conduction regimes have been analyzed and the evolution of the electronic mean free path (MFP) has been estimated, as functions of dopant density, nanotube radius, applied magnetic field, etc. [9-11]. Besides, the electronic structure of boron and nitrogen codoped nanotubes has been investigated, with special attention given to the variation of the electrostatic potential along the tube. It has been pointed out that electronic gaps could arise in specific situations [12].

Focusing on the electronic transport in boron- and nitrogensubstituted nanotube heterostructures, Khalfoun and coworkers reported first that the effect of the $\mathrm{B}$ and $\mathrm{N}$ elements on the transport properties is highly dependent on the relative position of the two doping species [13]. When the substitutional atoms are first nearest neighbors, the QBSs repel each other and the effect of the two dopants is annihilated, which preserves the transmission at $E_{F}$, whereas the carrier diffusion by each quasibound state is restored when the two dopants are spaced, resulting in a strong reduction of the electronic transmission properties. Second, in the case of nanodomains, they showed that the mutual interaction of the N- and B-induced QBSs results in a discrete spectrum of retrodiffusion states. The energy of the resulting QBSs induced by the shape and the nature of the defects or domains is then the key parameter driving the transmission function at $E_{F}$.

In this paper we present our theoretical investigations on the electronic transport properties of $\mathrm{BN}$-doped single-wall CNTs. All the structures have the $\mathrm{B}_{x} \mathrm{C}_{1-2 x} \mathrm{~N}_{x}$ stoichiometry, with $x$ the doping ratio. The model for the electronic structure is presented in Sec. II A. Section II B describes the techniques used to calculate the electronic transport and to extract the mean free path. We have investigated the evolution of the MFP as a function of radius and doping ratio for metallic $(n, n)$ and semiconducting $(n, 0)$ single-wall CNTs. Our results are presented in Sec. III. 


\section{THEORETICAL MODELS}

To describe the electronic properties of carbon nanotubes containing a selected density of combined BN defects, we need first a model for the electronic structure enabling a reliable description for a wide range of structures, with the same accuracy and electronic reference energy. On the other hand, we want to characterize electronic properties at the mesoscopic scale ( $\mu \mathrm{m}$ size), hence dealing with nanotubes containing up to $10^{6}$ atoms. Furthermore, to estimate how a given density of $\mathrm{BN}$ defects affects the transport, statistics on different configurations are needed. We then adopt a tight-binding (TB) model that provides a good balance between reliability and efficiency.

\section{A. Basis set, parametrization}

Up to now, most calculations at the mesoscopic scale have been carried out with the simplest tight-binding approaches, usually in an orthogonal basis set. That model gives a good description of the electronic structure for pristine nanotubes. To account for boron and nitrogen substitutions in the network, an electrostatic correction was added on the diagonal part of the $H$ matrix only $[8,9,11]$. That method is unfortunately restricted to the simplest situations for which the combined influence of boron and nitrogen can be neglected: low density of defects, pure $\mathrm{N}$ or B doping, etc.

With a very different approach, Lopez-Bezanilla et al. [14] have studied the transport in functionalized nanotubes, with a covalent link between the functional group and the nanotube wall. In this situation, the simplest TB approach is not reliable, mainly because $s p^{2}$ and $s p^{3}$ hybridizations coexist in the simulation cell. Model Hamiltonian and overlap matrices (expanded on an orbital atomic basis set) were directly constructed from the density functional theory (DFT) Hamiltonian and overlap matrices ( $H$ and $S$ ) obtained after the self consistent field treatment. This highly reliable approach preserves the accuracy of the $a b$ initio electronic description. This method has also been successfully applied by Khalfoun and coworkers to study the effect of combined $\mathrm{B}$ and $\mathrm{N}$ substitutions in nanotubes [13]. It is unfortunately not tractable in the present problem, since the heavy basis set employed in DFT (eight orbitals per atom with long-range hopping integral terms) leads to time-consuming calculations, which limit the statistical averages on $\mu \mathrm{m}$ length CNTs.

In our model, a nonorthogonal TB formalism, we adopt a basis set formed by a single $p_{\perp}$ atomic orbital per atom. The curvature of the nanotube is consequently neglected, because it is not an important feature for the electronic states in the vicinity of the Fermi level. ${ }^{1}$

The determination of the matrix elements of the Hamiltonian and overlap matrices $(H$ and $S)$ is made by fitting matrix elements coming from DFT calculations. Reference

\footnotetext{
${ }^{1}$ In the present work, we pay attention to armchair and zigzag nanotubes only. However, an extension of our model to study chiral $(n, m)$ nanotubes is possible by adding an angular dependance of the hopping elements (with respect to the nanotube axis) as shown in Ref. [15]. Such a model method allows us to predict the small gap opening in (quasi)metallic nanotubes.
}

DFT calculations were performed using the SIESTA code [16], with the LDA approximation for exchange and correlation [17] prior to the TB parametrization. Core electrons were treated by norm-conserving pseudopotentials [18] and a double- $\zeta s, p$ basis set was used to solve the valence electron eigenproblem. The basis set has been optimized using the simplex downhill method $[19,20]$. The unit cell is a graphene-like system with periodic boundary conditions made from 600 atoms, allowing us to reasonably reduce the Brillouin zone sampling to the $\Gamma$ point.

First the analysis of overlap matrix elements in DFT calculations indicate that the overlap matrix is significant up to the third neighbors for each element considered. It indicates that the hopping integrals also could be neglected after the third neighbors. For reference, we consider a pristine supercell and then address different doping situations perturbing the initial Hamiltonian $H^{0}$ obtained for a pure $\mathrm{C}$ system. Isolated $\mathrm{B}$ or $\mathrm{N}$ substitution first modifies the on-site energies of the neighboring carbon atoms $[8,13]$. This correction is fitted by a sum of three Gaussian functions. It can then be considered as a Coulombian potential perturbing the Hamiltonian $H^{0}$ previously set. Moreover, the presence of such a heteroatom in the network also affects the hopping integral elements. Assuming that the perturbative potential smoothly evolves on the range of one atomic orbital, we used a Hückel formulation to describe the influence of the dopant on nondiagonal Hamiltonian elements:

$$
H_{i j}=H_{i j}^{0}+\frac{\delta \epsilon_{i}+\delta \epsilon_{j}}{2} S_{i j},
$$

where $\delta \epsilon$ correspond to the on-site corrections, that is, the electrostatic potential induced by the dopant. The evolution of this perturbative potential is plotted in Fig. 1 as a function of the distance from the heteroatom. On the same figure, we report the correction induced on the hopping integral between $\mathrm{C}$ first neighbors as a function of the mean distance of these $\mathrm{C}$ to the dopant. This method is very tractable since all of the TB parameters only depend on the on-site energy correction.

When both $\mathrm{B}$ and $\mathrm{N}$ substitutions are present in the supercell, the perturbative potential induced on $\mathrm{C}$ atoms is well described by the sum of isolated corrections [13]. In this codoping situation, we also paid attention to the evolution of on-site energy of dopants perturbing each other. Figure 2 reports the evolution of the on-site correction for various $\mathrm{BN}$ substitution geometries. Our fits based on isolated B or N are in good agreement with the DFT calculations for all the pair geometries.

Small domains formed by a cycle of three B and three $\mathrm{N}$ atoms, denoted $\mathrm{B}_{3} \mathrm{~N}_{3}$ are also considered. Unfortunately, these smallest hexagonal BN domains cannot be described with the previous additive model, since the exchange part starts to be non-negligible. For this specific case, our analysis indicates that the perturbation induced onto the matrix elements on the surrounding $\mathrm{C}$ atoms affects the first neighbors only, as depicted in the Fig. 2(d). For that reason, the Hamiltonian elements are then directly taken from the DFT output in our parametrization. These two methods are reliable and can be compared since they refer to similar DFT calculations, performed at the same accuracy level. 

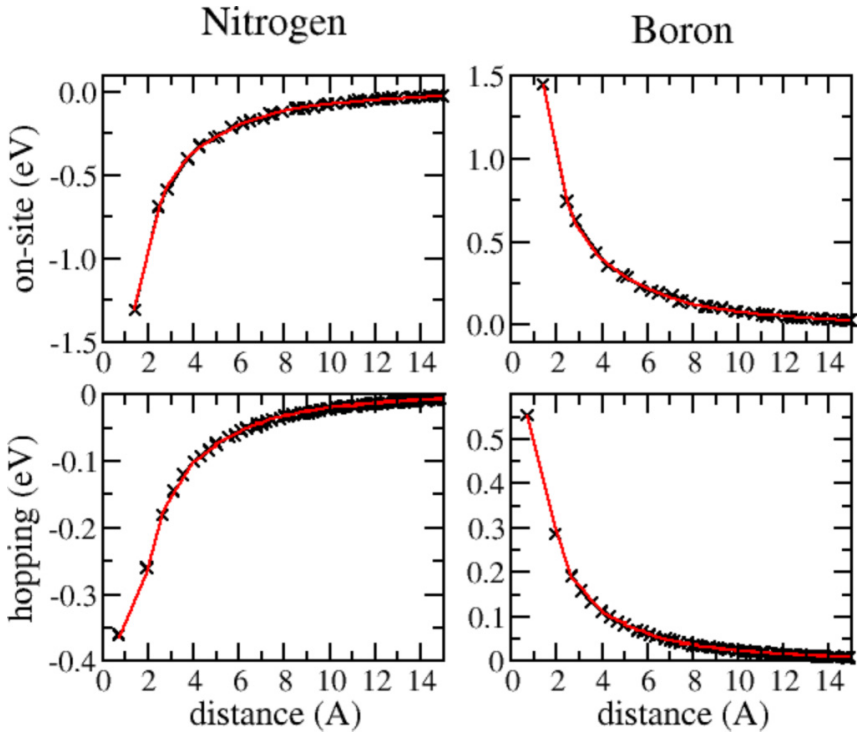

FIG. 1. (Color online) Evolution of corrections added to the C on-site energy as a function of the distance to the single atom dopant. The evolution of the first neighbors hopping integral between two $\mathrm{C}$ atoms are also plotted. The black points are the SIESTA matrix elements, and the solid lines correspond to the parametrization obtained by fitting the on-site energy by a sum of three Gaussians and with Eq. (1) for the hopping integrals.

\section{B. Electronic transport properties}

To calculate the conductance of our systems, we employed the recursive Green's function formalism in real space [21-23]
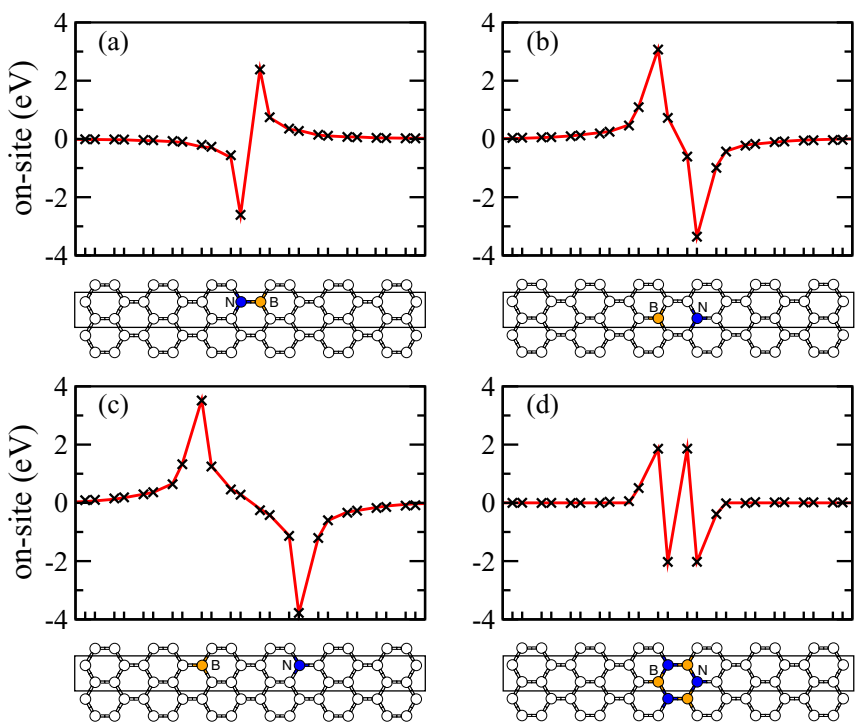

FIG. 2. (Color online) The evolution of the correction added to the on-site energy for different types of combined $\mathrm{B}$ and $\mathrm{N}$ substitutions along an armchair line (sites surrounded by the rectangle): (a) nearest neighbors $\mathrm{BN}$ pair, (b) $\mathrm{B}$ and $\mathrm{N}$ atoms separated by 2.48 $\AA$, (c) $\mathrm{B}$ and $\mathrm{N}$ atoms separated by $7.1 \AA$, and (d) a $\mathrm{B}_{3} \mathrm{~N}_{3}$ nanodomain. The black points are the directly read SIESTA matrix elements and the solid line corresponds the previously setup TB model. For the $\mathrm{B}_{3} \mathrm{~N}_{3}$ geometry, the TB model elements are not obtained by the fit, but directly taken from the SIESTA output. generalized to nonorthogonal basis sets [24]. This order $N$ approach allows us to study systems containing up to $10^{6}$ atoms. The system is divided into three parts: two semiinfinite pristine electrodes (labeled $L$ and $R$ ) connected to the channel region (the central part) with the condition that there is no direct tunneling Hamiltonian between the two electrodes. The projection of Green's function on the central region is

$$
G_{C}=\left(E S_{C}-H_{C}-\Sigma_{L}-\Sigma_{R}\right),
$$

where the $\Sigma$ terms are the self-energies corresponding to the coupling between the central part and the $L$ and $R$ electrodes. They write

$$
\Sigma_{X}=\left(E S_{C X}-H_{C X}\right) g_{X}\left(E S_{X C}-H_{X C}\right),
$$

where $X$ could be $R$ or $L$ and $g_{X}$ is Green's function for the isolated semi-infinite electrode. They are computed using the TB_Sim package [25], in which the recursive Green's function technique is implemented. The Landauer-Buttiker conductance [26] can then be evaluated from the transmission function

$$
T(E)=\operatorname{Tr}\left[\Gamma_{L} G_{C} \Gamma_{R} G_{C}^{\dagger}\right],
$$

where the $\Gamma$ terms are defined by

$$
\Gamma_{X}=i\left(\Sigma_{X}-\Sigma_{X}^{\dagger}\right)
$$

In this study the semi-infinite electrodes are treated as perfect nanotubes and the central part corresponds to the doped region. To investigate the influence of the $\mathrm{BN}$ substitutions on the transport properties in a large scale, we have computed the transmission function for a set of random distribution of defects. The mean conductance is determined by averaging over 200 configurations, assuming each one is equiprobable.

Figure 3(a) presents the transmission function of a pristine $(10,10)$ nanotube, a nanotube containing a single BN pair, and the average transmission for a $180-\mathrm{nm}$-long $(10,10)$ nanotube with a doping ratio of $0.1 \%$. From the transmission function, the MFP can be extracted from an interpolation between the quasiballistic and diffusive regimes [27]:

$$
T(L, E)=\frac{N(E)}{1+\frac{L}{\ell_{e}}},
$$
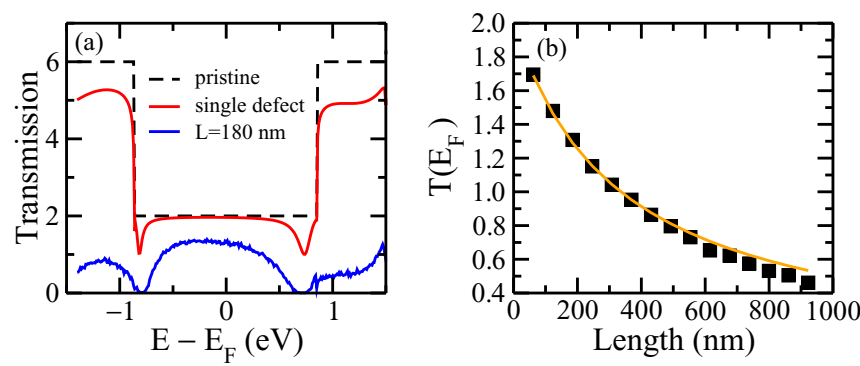

FIG. 3. (Color online) (a) The transmission function of a pristine $(10,10)$ nanotube (dashed, black), a $(10,10)$ nanotube containing a single BN-pair substitution (dotted, red), and one with a doping ratio of $0.1 \%$ and a length of $180 \mathrm{~nm}$ (solid, blue). (b) The transmission calculated at $E_{F}$ as a function of the length of the doped region (black squares). The solid line is obtained by fitting the calculated values with Eq. (6), which gives an estimation of $\ell_{e}=335 \mathrm{~nm}$ in this example. 


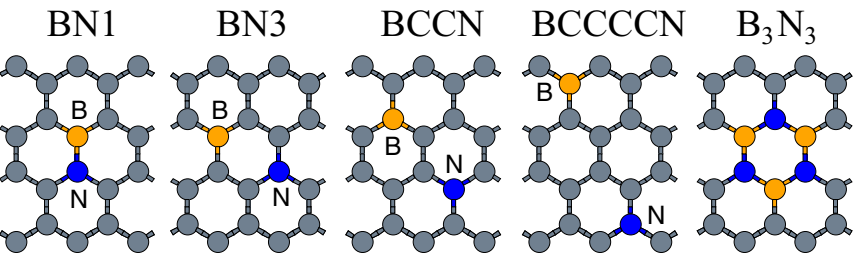

FIG. 4. (Color online) Different BN substitution geometries considered in this study. The distances between $\mathrm{B}$ and $\mathrm{N}$ for the pair cases are respectively $1.42,2.82,3.75$ and $6.15 \AA$.

with $N$ the number of accessible transmission channels in the similar pristine nanotube for the injection energy $E$. $L$ is the length of the doped region, and $l_{e}$ is the electronic MFP. This interpolation scheme is illustrated in Fig. 3(b). The calculated average transmission is plotted (squares) as a function of $L$ at the Fermi energy $E_{F}$. The MFP is extracted by fitting $T\left(L, E_{F}\right)$ with Eq. (6).

\section{RESULTS AND DISCUSSION}

We have considered five different $\mathrm{BN}$ geometries in the hexagonal network. Heteroatoms can be located as first or third neighbors, or separated by two or four carbons, as depicted on Fig. 4. We have investigated, for each geometry of BN substitutions the evolution of the transport properties as a function of the doping ratio and the radius for armchair nanotubes.

Figure 5(a) presents the evolution of the MFP for a $(10,10)$ nanotube doped with $0.1 \%$ of $\mathrm{B}$ and $\mathrm{N}$, as a function of the energy. The MFP is nearly zero in the vicinity of the first Van Hove singularity, close to the energy of the QBS induced by the B and $\mathrm{N}$ substitutions. Moreover, the MFP becomes very small for the energy ranges beyond the Van Hove singularity where the group velocity decreases drastically, as previously reported [28].
The MFP presents an inverse dependance with the density of defects $\ell_{e}=A / \rho$ and a linear evolution with the radius $\ell_{e}=\alpha R$, following the theoretical predictions based on Anderson-like disorder modeling [29]. The corresponding curves are respectively presented on Figs. 5(b) and 5(c). A relevant property is that the prefactors $A$ and $\alpha$ decrease when the $\mathrm{BN}$ distance increases, which is reminiscent of the energy scale of the QBS for the single diffusion center situation [13]. The scattering potential induced by the defect is softened when $\mathrm{B}$ and $\mathrm{N}$ atoms get closer, thus the quasibound states created by the BN defects are shifted away in the vicinity of the van Hove singularities. All these results indicate that the BN codoping behaves as a classical single diffusion center in the low doping limit. $^{2}$

Electronic transport in nanotubes containing BN domains also follows the previous laws concerning radius and concentration of defects. For that reason, the small domains $\mathrm{B}_{3} \mathrm{~N}_{3}$ can also be considered as point defects in the nanotube. On one hand, the induced scattering potential is spatially more extended than in the $\mathrm{BN}$ first-neighbors case and thus may affect the electronic transmission in a stronger manner. On the other hand, for a similar BN concentration the number of defects is three times lower than for the pair case. As a result the MFP for BN cycles is larger than for the BN pairs with the same BN doping ratio.

It is worth noticing that the energy formation for a given $\mathrm{BN}$ defect increases with the distance between the species. Besides, structural studies of $\mathrm{BN}$ nanodomains in $\mathrm{C}-\mathrm{BN}$ heterostructures indicate that the $\mathrm{BN}$ and $\mathrm{CC}$ bonds in the network are energetically favorable [30]. The system tends to maximize these kinds of bonds, leading to the formation of BN domains [31].

${ }^{2}$ When $\mathrm{B}$ and $\mathrm{N}$ substitutions get farther from each other, their combined effect is lost and the obtained mean free paths can be compared with those of a fully random distribution of $\mathrm{B}$ and/or $\mathrm{N}$ atoms, as in Refs. [9,11]. (a)

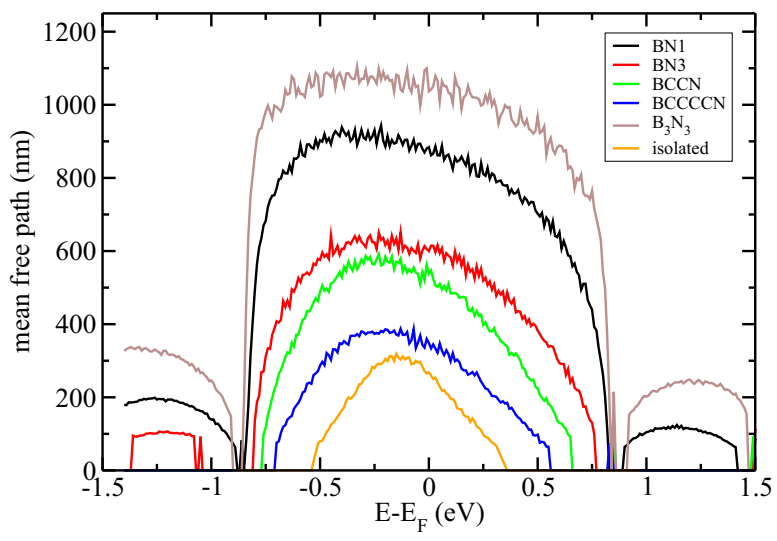

(b)

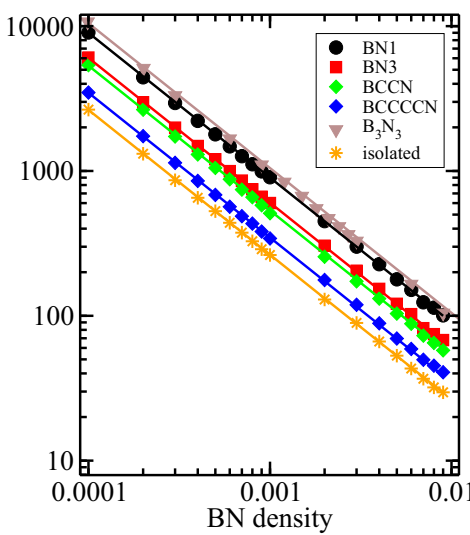

(c)

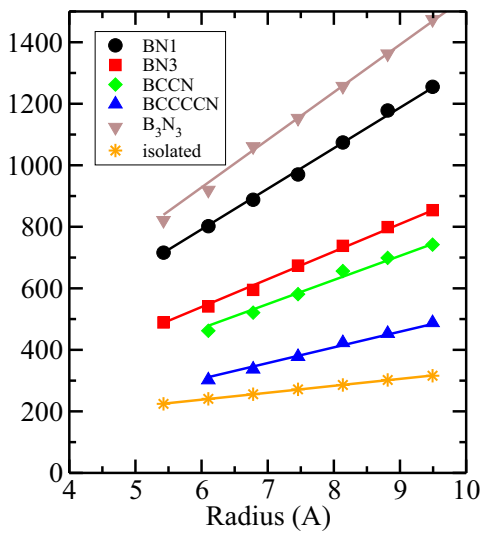

FIG. 5. (Color online) Transport properties in a BN-doped $(10,10)$ nanotube. (a) The evolution of the mean free path as a function of the injection energy, for the different BN defects sketched in Fig. 4. (b) Estimation of $\ell_{e}$ at the Fermi energy, as a function of the BN doping ratio. The full lines are obtained by fitting the evolution with $\ell_{e}=A / \rho$ law. The obtained value of $A$ are respectively $0.9,0.6,0.53$ and $0.35 \mathrm{~nm}$ for the pairs (with an increasing distance between B and N, as shown on Fig. 4) and $0.99 \mathrm{~nm}$ for the $\mathrm{B}_{3} \mathrm{~N}_{3}$ domain. (c) Estimation of the MFP at $E_{F}$, with a doping ratio of $0.1 \%$, as a function of the radius $R$. The full lines are obtained by fitting with a linear law $\ell_{E_{F}}=\alpha R$. For the pairs the $\alpha$ term is respectively estimated to $1320,900,750$, and 510, whereas we found $\alpha=1540$ for the domain. 
(a)

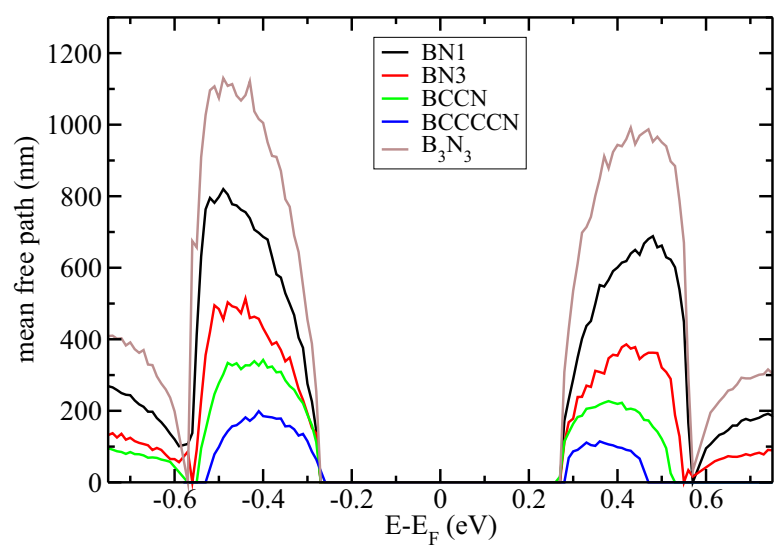

(b)

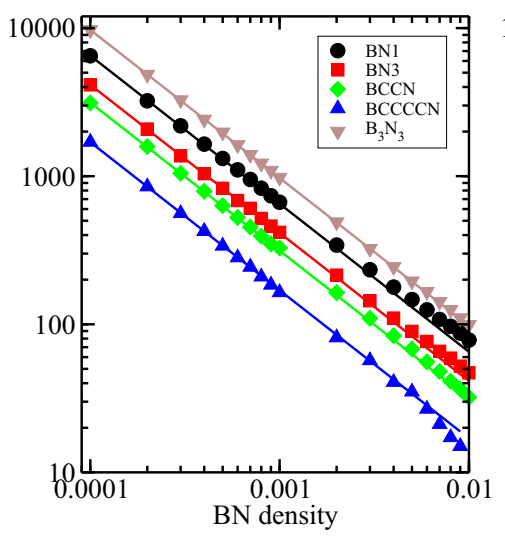

(c)

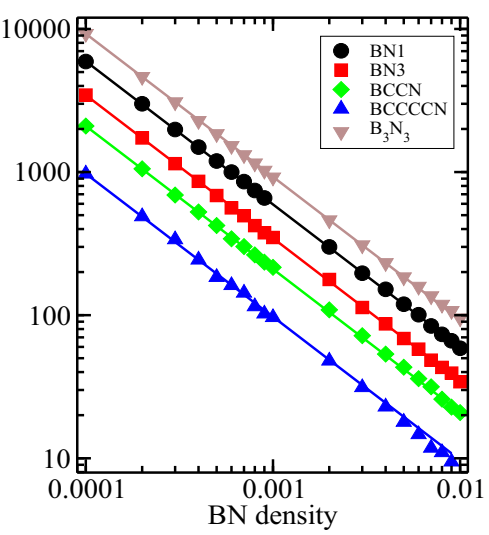

FIG. 6. (Color online) Transport properties in a BN-doped (19,0) nanotube. (a) Evolution of the mean free path as a function of the injection energy. (b) Mean-free-path estimate at $-0.4 \mathrm{eV}$ below the Fermi level as a function of the doping ratio for a $(19,0)$ nanotube. The full lines correspond to a fit by a $A / \rho$ law. The $A$ values are $0.65,0.41,0.31,0.17 \mathrm{~nm}$ for the BN pair and $0.97 \mathrm{~nm}$ for the cycle. (c) Same data and fits at $+0.4 \mathrm{eV}$ over the Fermi level. The $A$ values are $0.59,0.34,0.21,0.10 \mathrm{~nm}$ for the BN pair and $0.93 \mathrm{~nm}$ for the cycle.

It is interesting to point out that the transmission function presents an asymmetric behavior with respect to the electronic injection energy. Indeed, the transport properties are more affected for energies above the Fermi level than below. The maximum MFP value is reached for an energy around $0.25 \mathrm{eV}$ below the Fermi level. This feature is not intuitive when considering previous studies addressing single defects only. A single $\mathrm{BN}$ pair, or a $\mathrm{B}_{3} \mathrm{~N}_{3}$ cycle, seems to be transparent for incident electrons, and the transmission function is just affected around the Van Hove singularities [13]. Actually, the potential well induced by nitrogen is deeper than the potential hill induced by boron. As a consequence, the QBS induced by a single substitution of boron is closer to the first Van Hove singularity than the one coming from the nitrogen case, explaining this asymmetry in the MFP curve.

We have also paid attention to a semiconducting nanotube, with the zigzag symmetry. The results we obtained for a $(19,0)$ nanotube with a $0.1 \%$ doping ratio are presented on Fig. 6(a). The evolution of the MFP as a function of the energy indicates that this asymmetric behavior is still present for the semiconducting CNTs. Energy ranges corresponding roughly to the valence and conduction bands have been selected respectively to -0.4 and $+0.4 \mathrm{eV}$. The evolution of the MFP as a function of the doping ratio is depicted in Fig. 6(b) for an energy range corresponding to the valence band and Fig. 6(c) for the conducting band.

Again, the electronic mean free path is inversely proportional to the dopant density, and the prefactor decreases with the distance between the $\mathrm{B}$ and $\mathrm{N}$ atoms. For the energy range corresponding to the valence band state, a deviation to the $A / \rho$ law is observed for high dopant concentrations. This feature is related to a deformation of the band structure induced by the dopant that shifts the valence band. For the states in the conduction band, no deviation was observed, which suggests that the conduction band is less sensitive to the dopant than the valence band. The asymmetric behavior is also more pronounced for distant $\mathrm{BN}$ couples, two times larger for the valence band than for the conducting band. This asymmetry tends to disappear when the distance between $\mathrm{B}$ and $\mathrm{N}$ decreases as well as for small domains.

\section{SUMMARY}

In conclusion, we have investigated transport properties of carbon nanotubes with random distributions of $\mathrm{BN}$ pairs and small domains. The electronic mean free path decreases when doping species are dispersed, which could have important applications since the doping process in carbon nanotubes has a tendency to segregate $\mathrm{BN}$ into domains. We report that all considered $\mathrm{BN}$ geometries can be treated as classical point defects, and that the mean free path is inversely proportional to the doping ratio and proportional to the radius.

We also report an asymmetric behavior of the mean free path with injection energy. As a perspective it could be interesting to study the behavior of larger BN domains and then estimate the critical domain size over which one of the previous laws cannot be applied.

\section{ACKNOWLEDGMENTS}

Prof. Luc Henrard and Dr. Cyrille Barreteau are acknowledged for fruitful discussions about this work. This work was financed by the Nano-Simulation contract coming from the CEA Transversal Nano-Science Programme, and by the French National Research Agency (ANR) through the NANOSIMGRAPHENE Project No. ANR-09-NANO-016-01.
[1] M. Terrones, A. Souza, and A. Rao, in Carbon Nanotubes, Topic in Applied Physics, Vol. 111, edited by A. Jorio, G. Dresselhaus, and M. Dresselhaus (Springer, Berlin, 2008), p. 531.
[2] J. S. Lauret, R. Arenal, F. Ducastelle, A. Loiseau, M. Cau, B. Attal-Tretout, E. Rosencher, and L. Goux-Capes, Phys. Rev. Lett. 94, 037405 (2005). 
[3] R. Droppa, Jr., P. Hammer, A. C. M. Carvalho, M. C. dos Santos, and F. Alvarez, J. Non-Cryst. Solids 299-302, 874 (2002).

[4] M. Glerup, J. Steinmetz, D. Samaille, O. Stéphan, S. Enouz, A. Loiseau, S. Roth, and P. Bernier, Chem. Phys. Lett. 387, 193 (2004).

[5] E. Borowiak-Palen, T. Pichler, G. Fuentes, A. Graff, R. Kalenczuk, M. Knupfer, and J. Fink, Chem. Phys. Lett. 378, 516 (2003).

[6] S. Enouz, O. Stephan, J.-L. Cochon, C. Colliex, and A. Loiseau, Nanolett. 7, 1856 (2007).

[7] H. J. Choi, J. Ihm, S. G. Louie, and M. L. Cohen, Phys. Rev. Lett. 84, 2917 (2000).

[8] C. Adessi, S. Roche, and X. Blase, Phys. Rev. B 73, 125414 (2006).

[9] S. Latil, S. Roche, D. Mayou, and J.-C. Charlier, Phys. Rev. Lett. 92, 256805 (2004).

[10] S. Latil, F. Triozon, and S. Roche, Phys. Rev. Lett. 95, 126802 (2005).

[11] R. Avriller, S. Latil, F. Triozon, X. Blase, and S. Roche, Phys. Rev. B 74, 121406(R) (2006).

[12] P. E. Lammert, V. H. Crespi, and A. Rubio, Phys. Rev. Lett. 87, 136402 (2001).

[13] H. Khalfoun, P. Hermet, L. Henrard, and S. Latil, Phys. Rev. B 81, 193411 (2010).

[14] A. Lopez-Bezanilla, F. Triozon, S. Latil, X. Blase, and S. Roche, NanoLetters 9, 940 (2009).

[15] M. Damnjanović, T. Vuković, and I. Milošević, Solid State Commun. 116, 265 (2000).
[16] J. M. Soler, E. Artacho, J. D. Gale, A. Garcia, J. Junquera, P. Ordejon, and D. Sanchez-Portal, J. Phys.: Condens. Matter 14, 2745 (2002).

[17] J. P. Perdew and A. Zunger, Phys. Rev. B 23, 5048 (1981).

[18] N. Troullier and J. L. Martins, Phys. Rev. B 43, 1993 (1991).

[19] J. Junquera, O. Paz, D. Sánchez-Portal, and E. Artacho, Phys. Rev. B 64, 235111 (2001).

[20] E. Anglada, J. M. Soler, J. Junquera, and E. Artacho, Phys. Rev. B 66, 205101 (2002).

[21] M. P. López-Sancho, J. M. López-Sancho, and J. Rubio, J. Phys. F: Met. Phys. 14, 1205 (1984).

[22] R. Lake, G. Klimeck, C. Bowen, and D. Jovanovic, J. Appl. Phys. 81, 7845 (1997).

[23] A. Cresti, R. Farchioni, G. Grosso, and G. P. Parravicini, Phys. Rev. B 68, 075306 (2003).

[24] M. Buongiorno Nardelli, Phys. Rev. B 60, 7828 (1999).

[25] Y.-M. Niquet, http://inac.cea.fr/L_Sim/TB_Sim/.

[26] M. Büttiker, Y. Imry, R. Landauer, and S. Pinhas, Phys. Rev. B 31, 6207 (1985).

[27] R. Avriller, S. Roche, F. Triozon, X. Blase, and S. Latil, Mod. Phys. Lett. B 21, 1955 (2007).

[28] F. Triozon, S. Roche, A. Rubio, and D. Mayou, Phys. Rev. B 69, 121410 (2004).

[29] C. T. White and T. N. Todorov, Nature 393, 240 (1998).

[30] V. V. Ivanovskaya, A. Zobelli, O. Stéphan, P. R. Briddon, and C. Colliex, J. Phys. Chem. C 113, 16603 (2009).

[31] Z. Azevedo and R. De Paiva, Europhys. Lett. 75, 126 (2006). 